\title{
Is There A Systemic Inflammatory Effect of Cholesteatoma?
}

\author{
M. Mustafa Kılıçkaya ${ }^{1}$ Giray Aynali ${ }^{1} \quad$ Mustafa Tuz ${ }^{1}$ \\ ${ }^{1}$ Department of Otolaryngology, Suleyman Demirel University, \\ Isparta, Turkey \\ 2 Department of Medical Genetics, Suleyman Demirel University, \\ Isparta, Turkey \\ Int Arch Otorhinolaryngol 2017;21:42-45.
} \begin{abstract}
Otolaryngology, Medical Faculty of Suleyman Demirel University, 32260 Isparta, Turkey (e-mail: drmmkilic@gmail.com).

Özkan Bagcı
\end{abstract}

Abstract

Introduction Inflammation causes squamous epithelial transformation of the mucosa in the middle ear cavity and plays a role in the onset, growth, spread, and recurrence of cholesteatoma.

Objectives The objective of this study is to investigate the systemic inflammatory effect in chronic otitis with cholesteatoma.

Methods The study included a total of 311 patients comprising 156 patients with a pathology diagnosis of cholesteatoma and a control group of 155 with no active inflammation. The Neutrophil-to-lymphocyte Ratio (NLR) was calculated by dividing the neutrophil value by the lymphocyte value.

Results The mean NLR was $1.94 \pm 0.91$ in the patients with cholesteatoma and $1.94 \pm 0.85$ in the control group. We determined no statistically significant difference between the groups in respect of NLR $(p=0.983)$. We calculated the NLR as $2.01 \pm 1.00$ in patients with ossicle erosion and $1.82 \pm 0.69$ in those without ossicle erosion, $1.86 \pm 0.85$ in patients with bone erosion and $1.98 \pm 0.95$ in those without bone erosion. We determined no statistical difference between these values

\section{Keywords}

- otitis media

- cholesteatoma

- inflammation $(p=0.175)$.

Conclusion The results of this study showed that NLR had no predictive value in respect of bone erosions and associated complications in patients with cholesteatoma. The inflammatory effect of cholesteatoma is not systemic but remains more local.

\section{Introduction}

Cholesteatoma is a benign epithelial lesion, which destroys surrounding bone tissue as it grows and may lead to serious complications such as hearing loss, facial paralysis, vestibular disorders, and intracranial diseases. Clinically in cholesteatoma, there is chronic or recurrent purulent discharge. It has been reported that inflammation plays a role in the onset, growth, spread, and recurrence of cholesteatoma and that the hypoxic status of mastoid cells and the middle ear contributes to greater growth of the cholesteatoma tissue. ${ }^{1,2}$ In the squamous metaplasia theory of

received

April 6, 2016

accepted

May 4, 2016

published online

June 6, 2016 cholesteatoma, it has been reported that local inflammation causes squamous epithelial transformation of the mucosa in the middle ear cavity and that the inflammation induces epithelial proliferation. ${ }^{3}$

There is a relationship between cholesteatoma and the form of the immune response to inflammation (similar to that seen in wound healing). During inflammation, the matrix or perimatrix expresses cells such as monocytes, macrophages, and leukocytes and some angiogenetic factors such as, interleukin-8 (IL-8), cycloxygenase-2, and epidermal growth factor (EGF). ${ }^{4}$
Copyright $(2017$ by Thieme-Revinter

Publicações Ltda, Rio de Janeiro, Brazil
License terms

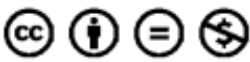


Peripheral neutrophils with lymphopenia, and therefore the increased NLR, has been recently accepted as a good marker of the response to inflammation in the body. Elevated NLR has been reported to be a poor prognostic factor in inflammation and some diseases such as obstructive sleep apnea syndrome, ischemic cardiac diseases, several types of cancer, and Bell's palsy. ${ }^{5-8}$

To the best of our knowledge, there has been no previous study in literature that has investigated the prognostic value of NLR in chronic otitis media with cholesteatoma. Therefore, this study is the first on this subject to investigate the relationship of NLR, which is used as a prognostic factor and inflammation marker in many diseases and inflammation, infection, and bone erosions found in chronic otitis media with cholesteatoma.

\section{Materials and Methods}

This retrospective study included a total of 311 patients, comprising 156 patients with a pathologic diagnosis of cholesteatoma and a control group of 155 . The study was conducted between January 2008 and February 2016. The Ethics board granted approval for this study in accordance with the Declaration of Helsinki. The patient group comprised 86 (55.1\%) men and 70 (44.9\%) women and the control group comprised 85 (54.8\%) men and 70 (45.2\%) women. We selected the control group subjects out of patients undergoing preoperative tests in the ear, nose, and throat polyclinic. When selecting the control group, the exclusion criteria were any active ear disease, acute inflammation, or infection which could affect the NLR, acute or chronic renal failure, heart disease, Bell's palsy, chronic liver disease, neoplasm, sleep apnea syndrome, chronic obstructive pulmonary disease, and neurological disorders. We obtained the neutrophil and lymphocyte values retrospectively from the patient files of the cholesteatoma patient group and the control group. We used high-resolution computed tomography (HRCT) in the radiological evaluation of patients with cholesteatoma. We recorded the preoperative HRCT findings and the intraoperative findings (facial canal, lateral semicircular canal-LSCC, scutum, tegmen, external auditory canal bone wall, ossicle erosion, etc.) of the patients with cholesteatoma.

\section{Haematological Analysis}

We collected blood samples into tubes containing calcium EDTA and used an automated blood cell counter for complete blood count (CBC) measurements (Beckman Coulter LH 780 Hematology Analyzer, USA). We calculated the NLR mathematically by dividing the neutrophil number by the lymphocyte number.

\section{Statistical Analysis}

In the statistical analysis of the study data, we used the $t$-test to evaluate the differences between the mean NLR of those with and without facial dehiscence, those with and without ossicle erosion, and those with and without bone erosion in the chronic otitis patients with cholesteatoma and the control group. We accepted a value of $p<0.05$ as statistically significant.

\section{Results}

The mean age of patients was $35.37 \pm 14.03$ years (range, 8-75 years) in the cholesteatoma patient group and $37.73 \pm 10.36$ years (range, 5-60 years) in control group. We calculated the mean NLR as $1.94 \pm 0.91$ in the patients with cholesteatoma and $1.94 \pm 0.85$ in the control group. We determined no statistically significant difference between the groups in respect of NLR $(p=0.983)$ (-Table 1).

We determined ossicle erosion in 102 (65.38\%) patients in the cholesteatoma group (-Fig. 1) and not in 54 (34.61\%). We calculated the NLR as $2.01 \pm 1.00$ in patients with ossicle erosion and $1.82 \pm 0.69$ in those without ossicle erosion. We determined no statistically significant difference in respect of NLR between those with or without ossicle erosion ( $p=0.175$ ).

We found bone erosions apart from the ossicles, such as in the facial canal, lateral semicircular canal (LSSC), scutum, promontorium, external auditory canal bone wall, tegmen, or sigmoid sinus at the rate of at least 1 in 57 (36.53\%) patients in the cholesteatoma patient group and not in 99 (63.46\%). The NLR was calculated as $1.86 \pm 0.85$ in patients with bone erosion and $1.98 \pm 0.95$ in those without bone erosion. We

Table 1 Mean NLR value of the patients with cholesteatoma and control group

\begin{tabular}{|c|c|c|c|c|}
\hline Group & $\mathbf{n}$ & Mean NLR value & Standard Deviation & $P$ value \\
\hline CSOM with Cholesteatom & 156 & 1.94 & \pm 0.91 & \multirow[t]{2}{*}{$p=0.983$} \\
\hline Control & 155 & 1.94 & \pm 0.85 & \\
\hline Ossicles erosion $(+)$ & 102 & 2.01 & \pm 1.00 & \multirow[t]{2}{*}{$p=0.175$} \\
\hline Ossicles erosion (-) & 54 & 1.82 & \pm 0.69 & \\
\hline Bone erosion $^{*}(+)$ & 57 & 1.86 & \pm 0.85 & \multirow[t]{2}{*}{$p=0.430$} \\
\hline Bone erosion* $^{*}(-)$ & 99 & 1.98 & \pm 0.95 & \\
\hline Facial canal dehiscence $(+)$ & 32 & 1.95 & \pm 0.81 & \multirow[t]{2}{*}{$p=0.982$} \\
\hline Facial canal dehiscence (-) & 124 & 1.94 & \pm 0.95 & \\
\hline
\end{tabular}

Abbreviations: CSOM, chronic suppurative otitis media; NLR, neutrophil-to-lymphocyte ratio.

*Apart from ossicle erosion, patients with erosion in at least one of the facial canal, semicircular canals, scutum, promontorium, external ear canal bone wall, tegmen, and sigmoid sinus. 


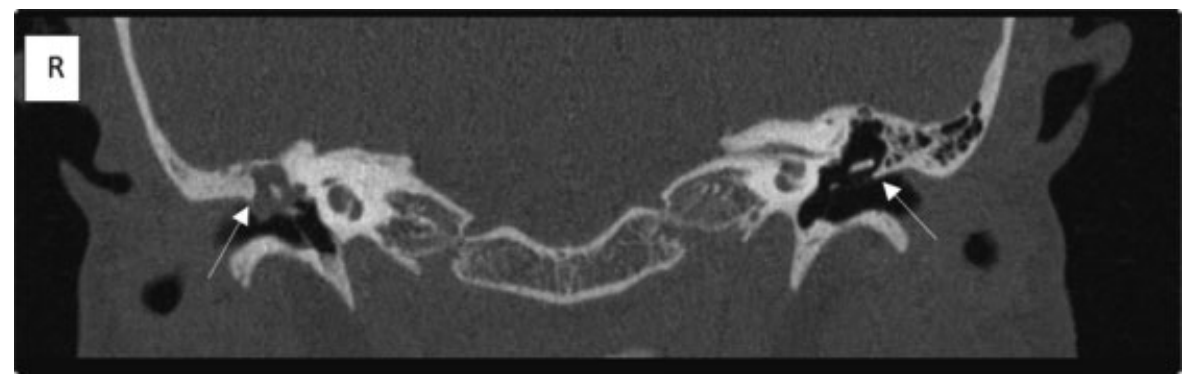

Fig. 1 The right scutum and ossicle erosion in patients with cholesteatoma. Arrow: scutum.

determined no statistically significant difference in respect of NLR between those with or without bone erosion $(p=0.430)$.

In the cholesteatoma patient group, we calculated the NLR as $1.95 \pm 0.81$ in patients with facial canal dehiscence $(n=32$, $20.51 \%)$ and as $1.94 \pm 0.95$ in those without facial canal dehiscence ( $n=124,79.48 \%$ ). We determined no statistically significant difference in respect of mean NLR between those with or without facial canal dehiscence $(p=0.982)$.

\section{Discussion}

Chronic otitis media with cholesteatoma leads to a chronic inflammatory process causing bone erosions localized in the middle ear and mastoid cavity. Histopathologically, cholesteatoma is benign but has locally aggressive behavior. Bone erosions may lead to meningitis, brain abscess, and facial paralysis. Bone erosion in cholesteatoma occurs with increased osteoclastic activation, which develops as a result of the mediators produced around the inflammatory event. Other factors causing bone erosion are the presence of keratin and inflammation with or without infection. ${ }^{9,10}$

Both gram (-) and gram ( + ) bacteria have been isolated in chronic otitis media with cholesteatoma and some inflammatory genes (such as nucleotide binding oligomerization domain-like receptor protein $3=$ NLRP3, Apoptosis-associated speck-like protein containing = ASC, and Caspase-1) have been found at a significantly high level in cholesteatoma tissue compared with normal tissue. These genes could be responsible in the etiopathogenesis. ${ }^{11}$

Chronic inflammation and bacterial infections activate epidermal hyperplasia of the tympanic membrane and contribute to the development of cholesteatoma by directly triggering the growth of the epidermal layer toward the middle ear cavity. Persistent inflammation causes the growth and aggressiveness of cholesteatoma. In patients with cholesteatoma, inflammatory mediators expressed by the mucosal innate immune system produce immune cells such as neutrophils, lymphocytes, and monocytes. The osteoblastic and osteoclastic activity balance is disrupted and bone erosion increases because of the mediators expressed from an infected cholesteatoma. Toll-like receptor (TLR) activation and the continued production of inflammatory cytokines play an important role in an osteoclastic event. In the perimatrix of acquired cholesteatoma, there is inflammatory cell infiltration such as lymphocyte and polymorphonuclear cells. $^{12-14}$
Acute inflammation is triggered by events such as bacterial or viral infections, trauma, tissue necrosis, and immune reactions. $\mathrm{T}$ lymphocytes play a greater role in the response of the body to viral infections. The basic leukocytes in acute infection are neutrophils. ${ }^{15}$ Clinically, the peripheral blood leukocyte count is closely related to inflammation in patients. An increase in leukocytes is reflected in the immune response of an individual to malignancy, inflammation and infection. It has been reported that a high NLR is related to a reduction in anti-tumor activity, progression of the tumor and metastasis. ${ }^{16}$ In several studies, an association has been found between a high NLR level and the prognosis and severity of disease in several cancers, such as oral squamous cell cancer, laryngeal squamous cell cancer, and gastric adenocarcinoma and in inflammatory diseases such as Bell's palsy and sudden hearing loss. ${ }^{16-19}$

In the current study, in which we examine the relationship between cholesteatoma occurring with bone erosion observed in a hypoxic middle ear and elevated NLR in bone erosion, tissue necrosis, and hypoxic conditions, we determined no significant difference in respect of NLR elevation between the patient and control group. There was also no significant difference in respect of NLR between those with and without bone erosion, ossicle erosion, or facial canal dehiscence. While an increase in NLR is expected in inflammatory diseases, the fact that there was no increase in NLR in cholesteatoma, including in cases with bone erosions, demonstrates that no systemic reaction is given by the organism to the formation of cholesteatoma. Even if the cholesteatoma was very aggressive, it was not seen to significantly increase the level of NLR.

\section{Conclusion}

Although NLR is related to the prognosis and severity of several diseases, we found no association with the progression and aggressiveness of cholesteatoma. However advanced cholesteatoma may be, it remains local and the organism is seen not to give a systemic response.

\section{References}

1 Louw L. Acquired cholesteatoma pathogenesis: stepwise explanations. J Laryngol Otol 2010;124(6):587-593

2 da Costa SS, Teixeira AR, Rosito LP. The contralateral ear in cholesteatoma. Eur Arch Otorhinolaryngol 2016;273(7): 1717-1721 
3 Lee JH, Hong SM, Kim CW, Park YH, Baek SH. Attic cholesteatoma with tiny retraction of pars flaccida. Auris Nasus Larynx 2015; 42(2):107-112

4 Kuo CL. Etiopathogenesis of acquired cholesteatoma: prominent theories and recent advances in biomolecular research. Laryngoscope 2015;125(1):234-240

5 Williams BA, Merhige ME. Association between neutrophil-lymphocyte ratio and impaired myocardial perfusion in patients with known or suspected coronary disease. Heart Lung 2013;42(6): 436-441

6 Bucak A, Ulu S, Oruc S, et al. Neutrophil-to-lymphocyte ratio as a novel-potential marker for predicting prognosis of Bell palsy. Laryngoscope 2014;124(7):1678-1681

7 Seretis C, Gourgiotis S, Gemenetzis G, Seretis F, Lagoudianakis E, Dimitrakopoulos G. The significance of neutrophil/lymphocyte ratio as a possible marker of underlying papillary microcarcinomas in thyroidal goiters: a pilot study. Am J Surg 2013;205(6): 691-696

8 Yenigun A, Karamanli H. Investigation of the relationship between neutrophil-to-lymphocyte ratio and obstructive sleep apnoea syndrome. J Laryngol Otol 2015;129(9):887-892

9 Chole RA, Hughes RM, Faddis BTJ. Keratin particle-induced osteolysis: a mouse model of inflammatory bone remodeling related to cholesteatoma. J Assoc Res Otolaryngol 2001;2(1):65-71

10 Ajalloueyan M. Experience with surgical management of cholesteatomas. Arch Otolaryngol Head Neck Surg 2006;132(9):931-933
11 Kariya S, Okano M, Zhao P, et al. Activation of NLRP3 inflammasome in human middle ear cholesteatoma and chronic otitis media. Acta Otolaryngol 2016;136(2):136-140

12 Louw L. Acquired cholesteatoma: summary of the cascade of molecular events. J Laryngol Otol 2013;127(6):542-549

13 Li N, Qin ZB. Inflammation-induced miR-802 promotes cell proliferation in cholesteatoma. Biotechnol Lett 2014;36(9):1753-1759

14 Si Y, Chen YB, Chen SJ, et al. TLR4 drives the pathogenesis of acquired cholesteatoma by promoting local inflammation and bone destruction. Sci Rep 2015;5:16683

15 Kumar V, Abbas AK, Aster JC. Robbins Basic Pathology. General pathology of infectious diseases. Philadelphia, PA: Elsevier; 2013: 309-326

16 Fang HY, Huang XY, Chien HT, et al. Refining the role of preoperative C-reactive protein by neutrophil/lymphocyte ratio in oral cavity squamous cell carcinoma. Laryngoscope 2013;123(11):2690-2699

17 Kum RO, Ozcan M, Baklaci D, et al. Investigation of neutrophil-tolymphocyte ratio and mean platelet volume in sudden hearing loss. Braz J Otorhinolaryngol 2015;81(6):636-641

18 Wong BY, Stafford ND, Green VL, Greenman J. Prognostic value of the neutrophil-to-lymphocyte ratio in patients with laryngeal squamous cell carcinoma. Head Neck 2016;38(Suppl 1): E1903-E1908

19 Graziosi L, Marino E, De Angelis V, Rebonato A, Cavazzoni E, Donini A. Prognostic value of preoperative neutrophils to lymphocytes ratio in patients resected for gastric cancer. Am J Surg 2015;209(2):333-337 